\title{
ON A FIELD-INDUCED SPIN-DENSITY WAVE IN BECHGAARD SALTS
}

\author{
M. Apostol \\ Department of Theoretical Pliysics, Institute of Atomic Pliysics \\ Magurele-Bucharest MG-6, P.O. Box MG-35, Romania \\ (Received February 1, 1994)
}

\begin{abstract}
A new type of field-induced spin-density wave is shown to occur in a weak-coupling layered model which may be relevant for the highly anisotropic electronic structure of the Becligaard salts. The modulation vector of this instability is perpendicular to the layers and close to the direction of the magnetic field applied perpendicular to the reciprocal layers. The magnitude of the modulation vector, order parameter and critical tempcrature have an oscillatory dependence on the magnitude of the magnetic field.
\end{abstract}

PACS numbers: $75.30 . \mathrm{Fv}, 75.50 . \mathrm{Rr}, 71.45 . \mathrm{Lr}$

\section{Introduction}

The Bechgaard salts (TMTSF) ${ }_{2} \mathrm{X}$ [1], where TMTSF stands for tetramethyl-tetraselenafulvalenium $\mathrm{C}_{10} \mathrm{H}_{12} \mathrm{Se}_{4}$ and $\mathrm{X}$ denotes an anion like $\mathrm{PF}_{6}, \mathrm{AsF}_{6}, \mathrm{ClO}_{4}$, etc., exhibit a great variety of electronic phases, depending on pressure and magnetic field. They crystallize in the triclinic system with the unit cell parameters $a=7.3 \AA, b=7.7 \AA$ and $d=13.5 \AA$, and the angles between the unit cell axes $\alpha=83^{\circ}, \beta=86^{\circ}$ and $\gamma=71^{\circ}$ [2]. The electronic structure is very anisotropic, and can be described in a first approximation by the energy band

$$
E=t_{a}\left(1-\cos k_{x} a\right)+t_{b}\left(1-\cos k_{y} b\right)+t_{c}\left(1-\cos k_{z} d\right),
$$

with the transfer integrals $t_{a}: t_{b}: \iota_{c} \approx 100: 10: 0.3$, the largest bandwidth $2 \iota_{a} \approx 0.7 \mathrm{eV}$ and one clectron per unit cell $[3,4]$. The wave vector components $k_{x, y, z}$ are directed along the reciprocal axes $a^{*}, b^{*}$ and, respectively, $d^{*}$. Since the transfer integral $t_{c}$ is much smaller than $t_{a}$ and $t_{b}$ we may consider these compounds as made of a succession of $a, b$-layers, periodically arranged and weakly coupled along the $d$-direction.

The Fermi sea corresponding to this electronic structure is completely filled along the $y$ - and $z$-directions, and the Fermi surface has a nesting vector $\left(2 k_{\mathrm{F}}, \pi / b\right.$, $\pi / d)$ with $2 k_{\mathrm{F}}=\pi / a$. Consequently, modulation instabilities may be expected to occur in these compounds [5]. Indeed, in the absence of the magnetic field it was 
found that some of them undergo a spin-density wave (SDW) transition below 10-20 K, while others exhibit a fluctuating charge-density wave, as driven by anion ordering; under moderate pressure $(\leq 15 \mathrm{kbar}$ ) most of these materials become superconducting below 2-3 K [6].

Anomalous behaviour has been reported at least for $\mathrm{X}=\mathrm{PF}_{6}, \mathrm{ClO}_{4}$ compounds when a moderate magnetic field $(\sim 10 \mathrm{~T})$ is applied approximately perpendicular to the layers. It includes Shubnikov-de Ilaas oscillations in the magnetoresistance [7], NMR magnetization [8], IIall-type effect [9, 10], and field-dependent anomalies in the electronic spccific heat [11]. The information gathered from such pieces of evidence has led to the suggestion that closed electronic orbits may exist in the $a^{*}, b^{*}$-plane in the presence of the magnetic field, and that a SDW may survive (providing the pressure is not very high) below $\sim 10 \mathrm{~K}$ and for certain values of the magnetic field above a threshold value of $\sim 2-3 \mathrm{~T}$.

Indeed, the SDW response in the magnetic ficld has been investigated within the quasi-classical approximation $[12,13]$, and field-induced instabilities have been found to persist, with the modulation vector close to that of the SDW occurring in the absence of the magnetic field. The reason for such a behaviour is that an imperfect nesting can partly be recovered for certain values of the magnetic field, due to the combined orbital and Zceman offects. Ilowever, the application of these results to the Bechgaard salts is questionable, since the quasi-classical approximation requires quasi-frec electron states, i.e. quasi-isotropic dependence of the energy on the wave vector, with relatively small wavelengths, while the electronic structure of the Bechgaard salts is highly anisotropic. On the other hand, it is difficult to reconcile the existence of closed electronic orbits and a :lesting vector of the Fermi surface in the plane perpendicular to the magnetic iseld.

IIere we provide an alternate description of a succession of field-induced SDW instabilities in a wcak-coupling layered model of the Becligaard salts. It is shown that the large anisotropy of the transfer integrals $t_{a}$ and $t_{b}$ leads to an effective-mass approximation (instead of the quasi-classical approximation) for the electron motion in the $a^{*}, b^{*}$-plane in the presence of the magnetic field applied along the $d$-axis. Solving for the encrgy eigenvalues we obtain a series of Landau bands, which do not overlap if the inter-layer coupling is weak enough (or the magnetic field is high). These Landau bands are one-dimensional, i.e. they only depend on the wave vector component $k_{z}$ along the $d^{*}$-direction (i.e. perpendicular to the layers). The Fermi "surface" of the partly filled top band reduces to $\pm k_{\mathrm{F}}$, where $k_{\mathrm{F}}$ is the Fermi wave vector. Consequently, modulation instabilities may occur along the direction perpendicular to the layers (and close to the direction of the magnetic field), whose $2 k_{\mathrm{F}}$-wave vector varies periodically with the magnitude of the magnetic ficld. The critical temperature and the order parameter of such a SDW instability are estimated and shown to have an oscillatory dependence on the magnitude of the magnetic field. 


\section{Landau energy bands}

We consider the motion of an electron in a crystalline potential which gives rise to the energy band (1), and simultaneously in a magnetic field directed along the $d$-axis. The corresponding vector potential can therefore be written as $A_{x}=-I y, A_{y}=A_{z}=0$, where $H$ is practically the magnitude of the applied magnetic field, as a consequence of the fact that the triclinic angles differ only slightly from the right angles. $A$ pplying a magnetic field the energy changes only little, while the symmetry of the Hamiltonian is reduced such as it has not any more the crystal periodicity. Consequently, whereas the crystalline energy bands are practically preserved, the Brillouin zones become meaningless in the presence of the magnetic field, and they may only be kept as a matter of convention. For low energy the electron motion in the plane perpendicular to the magnetic field is localized over distances of the order of the cyclotron length $\xi_{\mathrm{c}}$. Since this length is much larger than the lattice constants for practically all the available values of the magnetic field, it follows that a continuum-lattice approximation is valid for the electron motion in the $a^{*}, b^{*}$-plane as long as the corresponding energy does not exceed the crystalline energy. Usually, i.e. for quasi-isotropic electronic structures, this only holds for low values of the filling factor. Ilowever, in the case of highly anisotropic electronic structures the continuum-lattice approximation may also hold for rather high values of the filling factor. We shall show this by comparing the Fermi level $\mu$ of the continuum-lattice approximation with the Fermi level $\mu_{c}$ of the crystalline electronic structure in the case of the Bechgaard salts.

The continuum-lattice approximation for the energy band (1) in the $k_{x}, k_{y}$-plane reads

$$
E=\frac{1}{2} t_{a} a^{2} k_{x}^{2}+\frac{1}{2} \iota_{b} b^{2} k_{y}^{2}+t_{c}\left(1-\cos k_{z} d\right) .
$$

The corresponding Fermi level for half-filling is readily obtained as $\mu=\pi \sqrt{t_{a} t_{b}}$, where the small contribution of $t_{c} \approx 1 \mathrm{meV}$ has been neglected. Making use of $t_{a} \approx 0.35 \mathrm{eV}$ and $t_{b} \approx 35 \mathrm{meV}$ we get $\mu \approx 0.35 \mathrm{eV}$. The Fermi level $\mu_{\mathrm{c}}$ of the half-filled band (1) is obtained from

$$
\mu_{\mathrm{c}}=t_{a}[1-\cos (\pi / 2+\delta)]=t_{a}[1-\cos (\pi / 2-\delta)]+2 t_{b} ;
$$

it gives the modulation parameter of the Fermi surface $\sin \delta=t_{b} / t_{a} \approx 1 / 10$ and $\mu_{\mathrm{c}}=t_{a}+t_{b} \approx 0.38 \mathrm{eV}$ (the small contribution coming from $t_{c}$ being again neglected). One can see that $\mu<\mu_{\mathrm{c}}$ and the continuum-lattice approximation is applicable. We emphasize that this is a consequerice of the high anisotropy of the transfer integrals $t_{a}$ and $t_{b}$, the inequality $\pi \sqrt{t_{a} t_{b}}<t_{a}+t_{b}$ holding for $t_{b} / t_{a} \leq 0.13$. On the contrary, the quasi-classical approximation is not valid for such highly anisotropic electronic structures. Indeed, the open electronic orbits on the Fermi surface are almost straight lines in this case for the half-filling, and the magnitude of the electron wave vector varies by almost a reciprocal vector on moving along such an orbit. Consequently, the electron wavelength changes by an amount of the order of the lattice constants when the electron moves over distances of the order of the unit cell size, and the quasi-classical approximation is not applicable. 
The effective (Peicrls) IIamiltonian provided by the continuum-lattice approximation (2) in the presence of the magnetic field is given by

$$
\mathcal{H}=\frac{1}{2 m_{a}}\left(p_{x}-\frac{e}{c} H y\right)^{2}+\frac{1}{2 m_{b}} p_{y}^{2}+t_{c}\left(1-\cos k_{z} d\right)-2 \beta H \sigma,
$$

where $p_{x, y}$ are the corresponding components of the momentum, $\beta=e \hbar / 2 m c$ is the Bohr magneton, $\sigma= \pm 1 / 2$ is the spin projection and the effective masses $m_{a}=\hbar^{2} / a^{2} t_{a}$ and $m_{b}=\hbar^{2} / b^{2} t_{b}$ have been introduced. The crystal wave functions along the $z$-direction and the plane waves along the $x$-direction are therefore preserved, but the electron wave functions along the $y$-direction become those of a displaced harmonic oscillator. We note that the characteristic scale length of these wave functions is the cyclotron length $\xi_{\mathrm{c}}=\left(\phi_{0} b / \pi H a\right)^{1 / 2}\left(t_{b} / t_{a}\right)^{1 / 4}$, where; $\phi_{0}=h c / e=4.14 \times 10^{-7} \mathrm{~T} \cdot \mathrm{m}^{2}$ is the quanta of magnetic flux. The energy eigenvalues corresponding to the effectivc-mass IIamiltonian (4) are given by the Landau bands

$$
\mathcal{E}=2 \beta H\left[\frac{m}{m^{*}}(n+1 / 2)-\sigma\right]+t_{c}\left(1-\cos k_{z} d\right)
$$

where the effective mass $m^{*}=\left(m_{a} m_{b}\right)^{1 / 2}$ has becn introduced, and $n$ denotes the quantum number of the harmonic oscillator state. These energy bands depend only on the $k_{z}$-component of the wave vector, and correspond to closed electronic orbits in the $a^{*}, b^{*}$-plane. Conventionally, one may say that the Fermi sea shrinks around the central $z$-axis of the Brillouin zone to a set of cylindrical sheets which extend up to $k_{x} a=\sqrt{2 \pi}\left(t_{b} / t_{a}\right)^{1 / 4} \approx \sqrt{2}$ and $k_{y} b=\sqrt{2 \pi}\left(t_{a} / t_{b}\right)^{1 / 4} \approx \pi \sqrt{2}$. Therefore, the higher energy states of the crystal are depleted to the lower ones in the presence of the magnetic field in a highly anisotropic clectronic structure. We note that a similar conclusion has been reached in studying a closely related model of layered structure [14].

The effective mass $m^{*}=\left(m_{a} m_{b}\right)^{1 / 2}=h^{2} / a b\left(l_{a} l_{b}\right)^{1 / 2}$ is close to the free electron mass for the Bechgaard salts. Indeed, using $a=7.3 \AA, b=7.7 \AA$, $t_{a} \approx 0.35 \mathrm{eV}$ and $t_{b} \approx 35 \mathrm{meV}$ we get $m^{*} \approx 1.2 \mathrm{~m}$. Consequently, the average interband spacing obtained from $(5)$ is $\left(\mathrm{m} / \mathrm{m}^{*}\right) 2 \beta H=0.83 \times 2 \beta H$, and a Zeeman splitting occurs, of magnitude $\left(1-m / m^{*}\right) 2 \beta H=0.17 \times 2 \beta H$. Since this splitting is small in comparison with the interband spacing we may neglect it in a first approximation, which amounts to setting $m^{*}=m$. The energy bands (5) can be represented in this case by

$$
\mathcal{E}=2 \beta H l+t_{c}\left(1-\cos k_{z} d\right)
$$

where $l=0,1,2, \ldots$ One can see from (5) and (6) that the lowest band $l=0$ is filled with electron states having all spins up, while the upper bands $l=1,2, \ldots$ contain electron states with both spin orientations. The bands have also a "transverse" degeneracy, the number of states per unit area and per spin being $n_{x y}=H / \phi_{0}$. The total density of states for each spin orientation in each band is therefore $n_{0}=H / \phi_{0} d$.

We assume now that the energy bands (6) do not overlap, i.e. the transfer integral $t_{c}$ is as small, or the magnetic field $I$ is as high as $2 \beta H>2 t_{c}$. For a weakly-coupled layered structure the transfer integral $t_{c}$ can be as small as this condition be fulfilled for relatively low values of the magnetic field. Using the value 
$t_{c} \approx 1 \mathrm{meV}$ for the Bechgaard salts we get $I I \geq 15 \mathrm{~T}$. For the more realistic energy bands given by (5) the non-overlap condition reads $\left(\mathrm{m} / \mathrm{m}^{*}\right) 2 \beta H>2 \iota_{c}$, which leads to $H>\left(\phi_{0} / \pi a b\right)\left(t_{c} / \sqrt{t_{a} t_{b}}\right) \approx 20 \mathrm{~T}$. Ilowever, we note that the actual transfer integral $t_{c}$ given by the band structure calculations may, in fact, be smaller, which would reduce the rather high threshold field given above. It can also be reduced by higher values of the in-layer transfer integrals $t_{a}$ and $t_{b}$, which would also bring $m^{*}$ closer to $m$. We also note that variations in the band structure parameters may change these conditions, a circumstance which may explain the variability of the magnetic properties among the mombers of the Bechgaard salts family, as well as the disappearance of these properties by increasing pressure.

Assuming the non-overlap condition satisfied the energy bands (6) are fully occupied except the top one, which has therefore a Fermi wave vector $k_{F}$. The corresponding density of electron states with a given spin orientation is $\bar{n}=n_{x y} k_{F} / \pi=n_{0} \eta$, where we have introduccd the reduced Fermi wave vector $\eta=k_{\mathrm{F}} d / \pi, 0<\eta<1$. Now wc can estimate straiglitforwardly the band filling. If $n_{\mathrm{e}}$ is the electron density and the top band has the label $l(l \geq 1)$ we have

$$
n_{\mathrm{e}}=n_{0}+2(l-1) n_{0}+\bar{n}=(2 l-1+2 \eta) n_{0},
$$

whence

$$
\eta=\frac{1}{2}\left[\frac{n_{\mathrm{e}} \phi_{0} d}{H}-(2 l-1)\right]
$$

for

$$
\frac{1}{2 l+1}<H / n_{\mathrm{e}} \phi_{0} d<\frac{1}{2 l-1}
$$

such as to fulfill the condition $0<\eta<1$. It is easy to see that the Fermi level in the presence of the magnetic field is given by $\mu_{I I}=\left(\mathrm{m} / \mathrm{m}^{*}\right) 2 \beta H l$, or, using (9), $\mu_{H} \leq\left(m / m^{*}\right) \beta n_{\mathrm{e}} \phi_{0} d=\pi \sqrt{t_{a} \iota_{b}}=\mu$, i.e. it is slightly below the Fermi level of the continuum-lattice approximation.

We remark that the reduced Fermi wave vector $\eta$ given by $(8)$ and (9) varies periodically between 0 and 1 on varying the field. For subsequent use we also compute here the density of states per energy at one of the Fermi "surface" ends, and per spin

$$
g=\frac{1}{2} \frac{\mathrm{d} \bar{n}}{\mathrm{~d} \mathcal{E}}=\frac{1}{2 \pi} n_{x y}\left(\mathrm{~d} \mathcal{E} / \mathrm{d} k_{z}\right)_{k_{F}}^{-1}=\frac{I I}{2 \pi \phi_{0} t_{c} d} \frac{1}{\sin \pi \eta},
$$

which also varies periodically with the magnetic field.

\section{Field-induced SDW}

The one-dimensional Fermi "surface" of the partly filled top bands obtained in the preceding section may exhibit, in principle, various instabilities. We note that a charge-density wave has been pointed out in a similar one-dimensional model [15], in connection with the magnetoresistance anomalies in graphite [16]. We shall restrict ourselves to looking for a SDW instability, as brought about by a purely electronic interaction of Ilubbard type [17]. We shall also assume that there is only one partly filled top band, i.e. only one Fermi wave vector $k_{\mathrm{F}}$. As we 
have discussed above this assumption amounts to neglecting the Zeeman splitting and to the non-overlapping condition. The Zecman splitting is negligible as long as the effective mass $m^{*}$ is close to the frce electron mass $m$, as it seems to happen in the Bechgaard salts. We note that a modulation instability of a subband with polarized spins is simultaneously both a charge- and a spin-density wave. The non-overlapping condition discussed in the preceding section yields rather high values of the threshold magnetic field. However, it is easy to see that we can still have only one Fermi wave vector for certain lower values of the magnetic field. Indeed, if the bands overlap, two or more $k_{\mathrm{F}}$ 's may occur. At the beginning this happens for the reduced Fermi wave vector $\eta$ near zero or unity, so that for rather large regions inside the strips given by $(9)$ we still have only one Fermi wave vector. Leaving aside those regions where $H$ is near $n_{\mathrm{e}} \phi_{0} d(2 l \pm 1)^{-1}$ we may apply the results of the model even for lower values of $H$, providing that we limit ourselves to $\eta$ near $1 / 2$. However, as the overlap increases the regions where the Fermi "surface" breaks into many pieces are more and more frequent, and there appear many electron and hole pockets and a cascade of competing instabilities.

According to these assumptions we may write down a one-dimensional, single-band model Hamiltonian

$$
\mathcal{H}=\sum_{k \sigma} \varepsilon_{k} c_{k \sigma}^{+} c_{k \sigma}+\frac{\lambda}{2 V} \sum_{q k_{1} k_{2} \sigma} c_{k_{1}+q \sigma}^{+} c_{k_{1} \sigma} c_{k_{2}-q \bar{\sigma}}^{+} c_{k_{2} \tilde{\sigma}}
$$

where $\varepsilon_{k}=t_{c}(l-\cos k d), V$ is the volume of the sample, $\bar{\sigma}=-\sigma$ and $\lambda$ is the coupling strength. The interband matrix elements of the interaction have been left aside, and the "transverse" degeneracy is included in (11) as an overall factor. This Hamiltonian is relevant for each $l=1,2, \ldots$ band, except the lowest one $(l=0)$, where all the spins are parallel and the Hubbbard-type matrix elements vanish.

Although more general situations can be imagined we restrict ourselves to looking for a SDW order parameter

$$
\Delta=\frac{\lambda}{V} \sum_{k}\left\langle c^{+}{ }_{k_{\mathrm{F}}+k \sigma} c_{-k_{\mathrm{F}}+k \bar{\sigma}}\right\rangle
$$

which may be taken real and spin independent. Tlic spin density is then given by

$$
\left\langle\sigma_{x}(z)\right\rangle=\frac{2 \Delta}{\lambda} \cos \left(2 k_{\mathrm{F}} z\right)
$$

We assume that the critical temperature of this transition is low, so that we may leave aside the effects of various fluctuations and estimate it in the weak-coupling case of the mean-field approximation. As it is well known, the critical temperature is given in this case by $T_{\mathrm{c}}=1.13 \Delta E \exp (-1 / \lambda g)$, where $g$ is the density of states (10) and $\Delta E$ is the bandwidth. Similarly, the gap parameter is provided by $\Delta=$ $1.78 T_{\mathrm{c}}$. Since the bands are very narrow, we may approximate $\Delta E$ by $t_{c}(1-\cos \pi \eta)$ for $0<\eta<1 / 2$ (electron conduction) and $t_{c}(1+\cos \pi \eta$ ) for $1 / 2<\eta<1$ (hole conduction). We obtain finally

$$
T_{c}= \begin{cases}1.13 t_{c}(1-\cos \pi \eta) \mathrm{e}^{-\lambda_{0} \sin \pi \eta / H}, & 0<\eta<1 / 2, \\ 1.13 t_{c}(1+\cos \pi \eta) \mathrm{e}^{-\lambda_{0} \sin \pi \eta / H}, & 1 / 2<\eta<1\end{cases}
$$

where the reduced Fermi wave vector $\eta$ is given by (8) and (9) and $\lambda_{0}=2 \pi \phi_{0} t_{\mathrm{c}} d / \lambda$. 
As one can see from (14), the critical temperature has an oscillatory variation with the magnetic field. It vanishes for $H=n_{e} \phi_{0} d(2 l \pm 1)^{-1}$, and reaches a maximum value inside each of these regions. In order to cstimate the magnitude of the critical temperature we may take the envelope of (14) for $\eta=1 / 2$

$$
\bar{T}_{\mathrm{c}}=1.13 t_{c} \mathrm{e}^{-\lambda_{0} / I I} \text {. }
$$

We note that a similar $H$-dependence of the critical temperature has previously been used for the experimental data in some of the Becligaard salts [4].

The critical temperature given by (15) is fitted to the experimental data given in Refs. [4] and [10] for $t_{c}=1 \mathrm{meV}$, which coincides with the result of the band structure calculations, and $\lambda_{0}=8 \mathrm{~T}$; for these values $\bar{T}_{\mathrm{c}}=3 \mathrm{~K}$ for $H=10 \mathrm{~T}$. Using $\lambda_{0}$ given below (14) we obtain a coupling strength of our model $\lambda=10^{3} \mathrm{eV} \cdot \AA^{3}$. For $H=10 \mathrm{~T}$ we also find from (9) that the highest occupied band has $l \approx 350$, and the width of the region over which the critical temperature is non-vanishing is $\sim 0.3 \mathrm{kOe}\left(n_{\mathrm{e}} \phi_{0} d \sim 7 \times 10^{3} \mathrm{~T}\right)$.

\section{Concluding remarks}

In conclusion one may say that an example has been given of a field-induced SDW in a weak-coupling layered model which may be relevant for the Bechgaard salts. The highly anisotropic electronic structure of the model allows three particular conditions to be satisfied. First, the large difference between the in-layer transfer integrals $t_{a}$ and $t_{b}$ leads to an effective-mass approximation for the electron motion in the magnetic field, such as to minimize the energy. Consequently, one-dimensional Landau energy bands are obtained, which amounts to closed electronic orbits in the plane perpendicular to the magnetic field and crystal wave functions along the direction perpendicular to the layers (and close to the direction of the magnetic field). Secondly, the effective mass is close to the free electron mass, such that the Zeeman splitting of the bands may be neglected. And thirdly, the interlayer coupling provided by the transfer integral $t_{c}$ is so weak that the bands do not overlap even for relatively low values of the magnetic field. Under these circumstances there is only one partly filled top band, whose one-dimensional Fermi "surface" may undergo modulation instabilities. The modulation vector of such an instability is perpendicular to the layers and its magnitude varies periodically with the magnitude of the magnetic field. The critical temperature and the gap parameter have been estimated for a SDW and shown to have an oscillatory dependence on the magılitude of the magnetic field. It has also been shown that the critical temperature of this model can reproduce satisfactorily the experimental data obtained for some Bechgaard salts from the indirect evidence of the IIall effect.

\section{References}

[1] K. Bechgaard, C.S. Jacobsen, K. Martensen, II.J. Pedersen, N. Thorup, Solid State Commun. 33, 1119 (1980).

[2] N. Thorup, G. Rindorf, II. Soling, K. Bechgaard, Acla Crystallogr. B 37, 1236 (1981). 
[3] P.M. Grant, Pliys. Rev. B 26, 6888 (1982).

[4] R.L. Greene, P.M. Chaikin, Physica B 126, 131 (1984).

[5] B. Horovitz, II. Gutfreund, M. Weger, Phys. Rev. B 12, 3174 (1975).

[6] For a review see, for example, J. Phys. (France) C3 44, (1983).

[7] J. Kwak, J. Schirber, R. Greenc, E. Engler, Phys. Rev. Lett. 46, 1296 (1981).

[8] L. Azevedo, J. Schirber, R. Greene, E. Engler, Physica B 108, 1183 (1981).

[9] M. Ribault, D. Jerome, J. Tuchendler, C. Weyl, K. Bechgaard, J. Phys. (France) 44, L953 (1983).

[10] P.M. Chaikin, M.Y. Choi, J.F. Kwak, J.S. Brooks, K.P. Martin, M.J. Naughton, E.M. Engler, R.L. Greene, Phys. Rev. Lell. 51, 2333 (1983).

[11] R. Brusetti, P. Garoche, K. Bechgaard, J. Phys. (France) C3 44, 1051 (1983).

[12] L.P. Gorkov, A.G. Lebed, J. Plys. (France) 45, L433 (1984).

[13] M. Heritier, G. Montambaux, P. Lederer, J. Phys. (France) 45, L943 (1984).

[14] P.M. Chaikin, T. Holstcin, M.Y. Azbcl, Philos. Mag. B 48, 457 (1983).

[15] D. Yoshioka, II. Fukuyama, J. Phys. Soc. Jpn. 50, L725 (1981).

[16] Y. Iye, P.M. Tedrow, G. Timp, M. Shayegan, M.S. Dresselhaus, G. Dresselhaus, A. Furakawa, S. Tanuma, Phys. Rev. B 25, 5478 (1982).

[17] K. Yamaji, J. Phys. Soc. Jpn. 51, 2787 (1982). 